\title{
Distortion of a polycrystalline Al bar in a vice fixture: molecular dynamics analysis of grain movement and rotation
}

\author{
Vardan Hoviki Vardanyan ${ }^{1} \cdot$ Barbara S. Linke ${ }^{2} \cdot$ Herbert M. Urbassek $^{1}$
}

Received: 16 March 2021 / Accepted: 5 July 2021 / Published online: 27 July 2021

(C) The Author(s) 2021

\begin{abstract}
The deformation of a nano-sized polycrystalline Al bar under the action of vice plates is studied using molecular dynamics simulation. Two grain sizes are considered, fine-grained and coarse-grained. Deformation in the fine-grained sample is mainly caused by grain-boundary processes which induce grain displacement and rotation. Deformation in the coarsegrained sample is caused by grain-boundary processes and dislocation plasticity. The sample distortion manifests itself by the center-of-mass motion of the grains. Grain rotation is responsible for surface roughening after the loading process. While the plastic deformation is caused by the loading process, grain rearrangements under load release also contribute considerably to the final sample distortion.
\end{abstract}

Keywords Nanofabrication $\cdot$ Distortion $\cdot$ Plasticity $\cdot$ Grain boundary $\cdot$ Grain rotation $\cdot$ Molecular dynamics

\section{Introduction}

The plastic deformation of polycrystalline metal samples is a topic of long-standing interest in materials science [1]. It is of immediate importance in the field of mechanical engineering, where plastic deformation processes are involved in the treatment of a workpiece, leading both to residual stresses and to workpiece distortions [2]. The origin and prediction of such distortions are the topic of scientific research [3-7]. To this date, it is still industrial practice to control distortions by trial-and-error tests or repositioning and reclamping.

While machining processes on the microscale are commonly used in ultra-precision engineering [8], nanometersized mechanisms are out of reach for most applications. However, in the fields of photolithography, chemical etching, laser machining, atomic force lithography, or

Herbert M. Urbassek

urbassek@rhrk.uni-kl.de; http://www.physik.uni-kl.de/urbassek/

1 Physics Department and Research Center OPTIMAS, University Kaiserslautern, Erwin-Schrödinger-Straße, D-67663 Kaiserslautern, Germany

2 Mechanical and Aerospace Engineering Department, University of California Davis, 1 Shields Ave, Davis, CA 95616, USA focused ion beam lithography, nanofabrication techniques are already being used [9]. Due to its significant future potential, the theory of nanocutting must be systematically developed [10]. It is therefore of interest to widen the understanding of plastic processes by including processes occurring in nanofabrication. Here, atomistic simulation methods based on molecular dynamics simulation, have proven useful $[11,12]$. The behavior of samples under homogeneous strain has been investigated best [13-20]. Also several machining processes have been studied, including nanoindentation [21, 22], scratching [23] and nanocutting [24] of surfaces. Several of the results obtained from these studies allowed extension to larger spatial scales $(\mu \mathrm{m}$-sized samples) for instance the generation of dislocations [22] or the effect of the tip geometry on the plastic processes [23]. Mono- and polycrystalline materials will behave differently in nanomanufacturing processes. For example for microcutting, Shimada et al. [25] used molecular dynamics simulation to show that a worksurface free of residual distortion can be obtained for monocrystalline copper, but nanometrically distorted layers remain on polycrystalline copper worksurfaces.

On the nanoscale, distortion and the generation of residual stresses are based on the plastic processes occurring in the material under mechanical loading. In many metals, plasticity is dominated by dislocations which are activated during loading $[1,26]$. In nanocrystalline material, dislocations and grain-boundary-based processes - in particular 
grain-boundary displacement and rotation - contribute to the plastic deformation [27-30]. The combination of dislocation and grain-boundary based processes and how they affect distortion has not been investigated up to now.

In the present paper, we will study the mechanical deformation of an $\mathrm{Al}$ bar which is compressed in an inhomogeneous way by vice plates [31] (see Fig. 1a). In addition to the plastic deformation, we investigate the processes occurring in the individual grains (i.e., grain displacement and rotation) and the distortion of the bar.

\section{Method}

We study an Al block (see Fig. 1a) with sizes of $80 \mathrm{~nm}$ $\times 40 \mathrm{~nm} \times 60 \mathrm{~nm}$ in $x, y$ and $z$ directions, consisting of approximately $12 \times 10^{6}$ atoms. The block is subject to periodic boundary conditions in the $y$ direction; the other surfaces are free. The bottom half of the $x-z$ surfaces are held by rigid vice plates. In the course of the simulation, these plates move inward along the $x$ direction with a velocity of $10 \mathrm{~m} / \mathrm{s}$ for $200 \mathrm{ps}$, compressing the $\mathrm{Al}$ bar from 80 to $78 \mathrm{~nm}$; then the fixtures are held for 200 ps to allow the sample to relax; and finally the vices are released by moving with $10 \mathrm{~m} / \mathrm{s}$ to their initial positions.

In a previous publication [31], this procedure was applied to a single-crystalline Al block where all surfaces were oriented in $\{100\}$ directions. In the present work, however, we use polycrystalline $\mathrm{Al}$ blocks. In order to understand the effect of grain size, we use two samples (see Fig. 1b): a finegrained sample consisting of 180 grains with an average size of $10 \mathrm{~nm}$, and a coarse-grained sample with 18 grains of $22 \mathrm{~nm}$ size. The small grain size is compatible with the nanoscale dimensions of the sample and is around two orders of magnitude below the grain size encountered in macroscale machining $(\sim 1 \mu \mathrm{m})$. The samples are generated using Voronoi tesselation [32-34]. For the preparation of relaxed samples, first, the samples are heated to $500 \mathrm{~K}$ for $50 \mathrm{ps}$ in order to relax the grain boundaries in the samples; then the temperature is kept constant for $50 \mathrm{ps}$, and finally the temperature is decreased to $300 \mathrm{~K}$ for another $50 \mathrm{ps}$. In a final step, the samples are relaxed for $250 \mathrm{ps}$ at 300 $\mathrm{K}$ in an isothermal-isobaric NPT ensemble such that all components of the stress tensor decrease to zero. During the compression and release simulations, the atoms in a stripe of width $1 \mathrm{~nm}$ in the middle part of the sample between the vice plates are subjected to a Nose-Hoover thermostat to keep the temperature at $300 \mathrm{~K}$. In addition, the lower part of this stripe with a height of $1 \mathrm{~nm}$ has been fixed in order to prevent rigid-body translation and rotation of the entire sample during the release phase.

The vice plates have a thickness of $2 \mathrm{~nm}$ and a height of $34 \mathrm{~nm}$. They consist of around 500,000 carbon atoms each, arranged in a rigid diamond-lattice structure.

The $\mathrm{Al}$ atoms interact via the Mendelev et al. potential [35] with each other. The Al-carbon interaction is modeled by a purely repulsive potential obtained from a LennardJones potential cut off at its minimum [31].

The simulations are performed with the software LAMMPS [36] and the results are analyzed and visualized using OVITO [37].

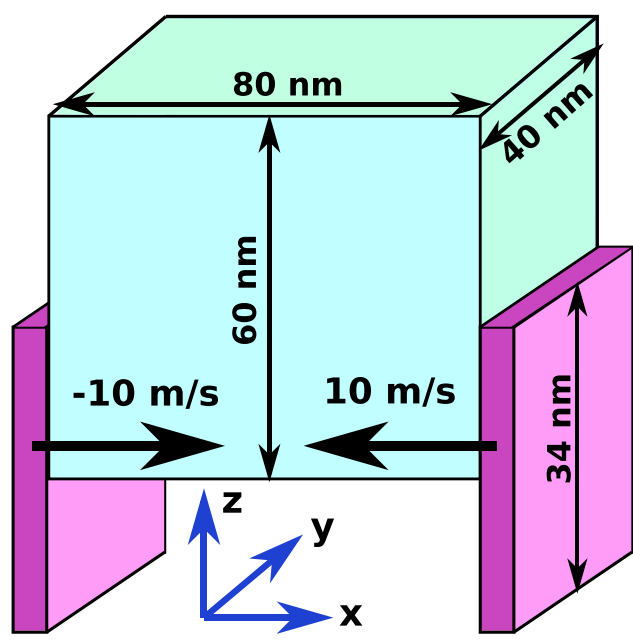

(a)

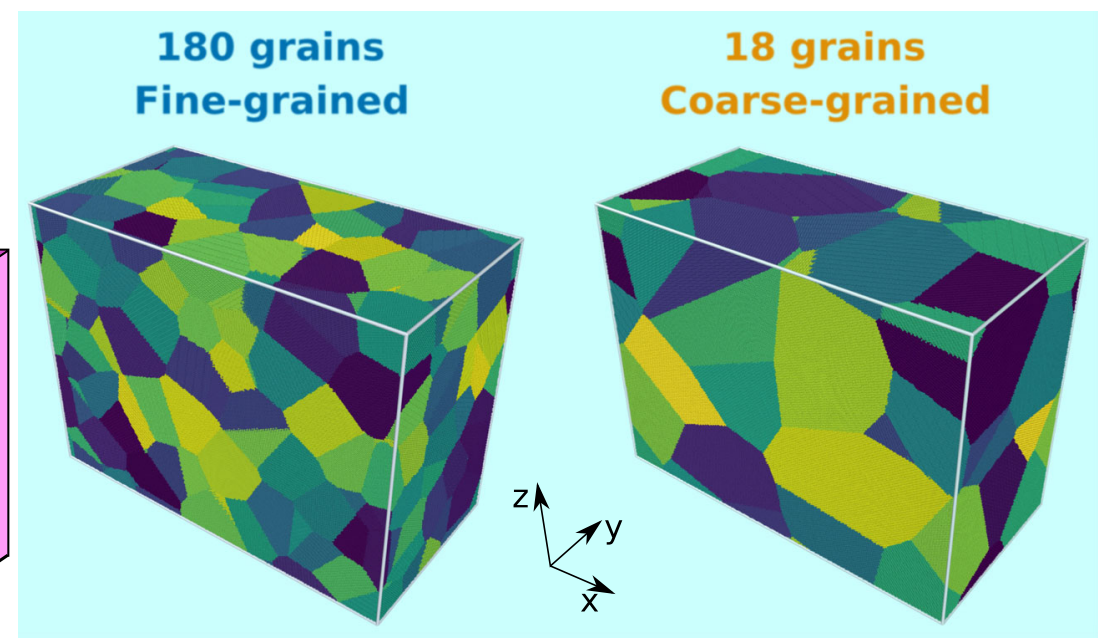

(b)

Fig. 1 a Schematic setup of the simulation system. The vice plates compress the Al bar laterally. b Two simulation samples containing 180 and 18 grains with average grains sizes of $10 \mathrm{~nm}$ and $22 \mathrm{~nm}$, respectively 


\section{Results}

\subsection{Forces}

The force on the vices during the pressing, holding and release phases of the simulation are shown in Fig. 2. We show the forces acting on the vice plates on the leftand right-hand side, in order to be able to discuss the asymmetry caused by grain displacements and rotations (see Sections 3.4 and 3.5 below).

During the pressing phase, initially the force increases in proportion to the distance moved by the plates; however, in the second half of the pressing phase, the forces saturate and even start decreasing. This happens because the yield stress of the material has been reached. The average pressure can be estimated as $p=F / A$; with the maximum force given by $F=3 \mu \mathrm{N}$ and the area of the vice plates given by $A=1200 \mathrm{~nm}^{2}$, the pressure is $p=2.5 \mathrm{GPa}$. This value may be compared to the hardness of single-crystalline $\mathrm{Al}$, which amounts to $6 \mathrm{GPa}$ for the interatomic interaction potential that we employ [38]. The yield stress in nanocrystalline samples has been found to be smaller and is in the range of 1.6 to $3.5 \mathrm{GPa}$, a value which varies with grain size [15, 39-43]. For the interatomic interaction potential used in the present work, a yield stress of 3.5-4.9 GPa was reported for nanocrystalline samples [44]. These values are considerably above the yield stress of macroscopic polycrystalline $\mathrm{Al}$ due to the Hall-Petch effect.

The yield mechanisms in the coarse- and fine-grained samples are different [41, 45], see discussion in Section 3.2 below, and hence the two samples have two different force maxima.

During the holding phase, the force decreases as the material relaxes internally by dislocation- and grainboundary-based mechanisms (see Section 3.2 below). Stress release is particularly pronounced for the coarse-grained sample, as here dislocations are more active. The dislocation

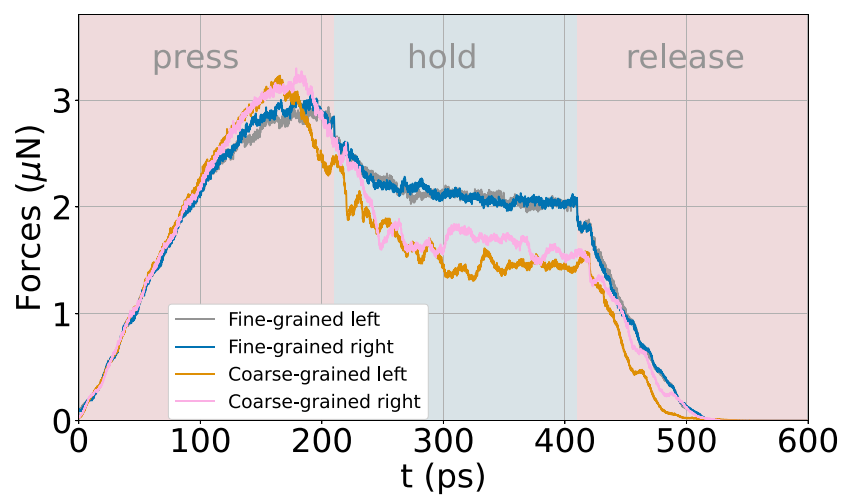

Fig. 2 Time evolution of the forces acting in $x$ direction on the vices activity is visible in the force evolution by the considerable fluctuations seen that originate from individual dislocation processes occurring in the sample. After around 100-150 ps of holding time, the forces have reached their equilibrium values. The final pressure reached in the coarse-grained sample is only half of that seen during the pressing phase and amounts to only $1.25 \mathrm{GPa}$.

During release of the vices, the force continuously decreases towards zero. The vices lose contact with the sample after a time of $500 \mathrm{ps}$, indicating that both samples have shrunk in $x$ direction by around $1 \mathrm{~nm}$ due to the plastic deformation in the entire compression and release operation.

We note that the strain rate used in the simulations amounts to $2.5 \times 10^{8} \mathrm{~s}^{-1}$. It has been shown [46, 47] that below strain rates of around $1 \times 10^{9} \mathrm{~s}^{-1}$, elastic and plastic properties - in particular the yield stress — depend only negligibly on strain rate. Above that critical strain rate, dislocation nucleation is prohibited.

\subsection{Deformation mechanism}

The deformation mechanisms of nanocrystalline materials are known to depend on grain size. In particular, it has been found [47] for fcc $\mathrm{Cu}$ that above a grain size of around 15 $\mathrm{nm}$, deformation is governed by dislocation plasticity, which occurs inside grains, and below a grain size of around 15 $\mathrm{nm}$, deformation is governed by grain-boundary processes, such as grain displacement and rotation. Dislocations nucleate in grains as a result of stress concentration at grain boundaries, while the stress in the grain interior is relieved for sufficiently large grains. While the quoted study [47] was on $\mathrm{Cu}$, the grain sizes chosen for this study -10 and $22 \mathrm{~nm}-$ lie clearly below and above the critical grain size, such that we expect to find these distinct behaviors also in $\mathrm{Al}$.

In order to quantify the deformation processes both inside the grains and in the grain boundaries, we use the von Mises shear strain (VMSS) that is readily evaluated for each atom in the sample [48]. Shear strain is the prime agent to nucleate and move dislocations, as well as to shape grain boundaries.

Focusing on the grain-boundary activity first, Fig. 3 shows the fraction of grain-boundary atoms with VMSS $>0.1$. The strain at the end of each phase (pressing, holding, and release) is calculated with respect to the configuration at the beginning of the phase. In the finegrained sample, in each phase more than half of the grain-boundary atoms have been considerably sheared, evidencing a high occurrence of grain-boundary mediated plastic deformation. The fraction of grain-boundary atoms that have been similarly sheared is considerably smaller for the coarse-grained sample. These numbers show that the 


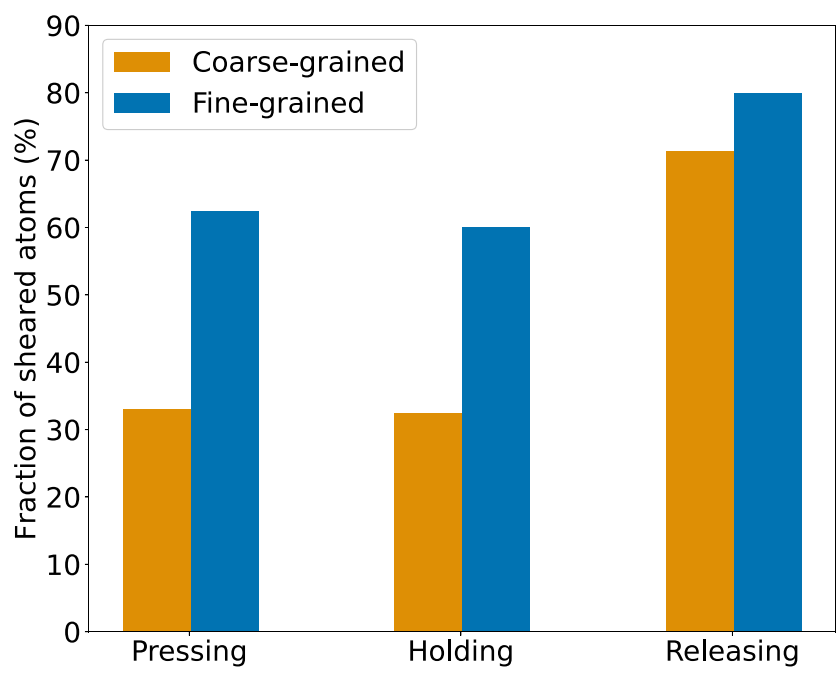

Fig. 3 Fraction of grain-boundary atoms with VMSS $>0.1$. The reference configurations for holding and releasing are the end of the pressing and the holding phase, respectively

above distinction made for nanocrystalline $\mathrm{Cu}$ is also true for machined Al. Interestingly, the fraction of sheared grainboundary atoms is largest under the release step. i.e., when the sample is allowed to return to a pressure-free state.

Apart from these fractions, the snapshots shown in Fig. 4 demonstrate the occurrence of dislocation-based and grainboundary-mediated processes in greater detail. In this view on the surface of the sample, dislocations inside the grains are marked and grain boundaries are colored according to whether their shear exceeded VMSS $=0.1$. An analysis of the generated dislocations using OVITO [37] shows that these mostly have Burgers vectors of $1 / 6\langle 112\rangle$ and $1 / 2\langle 110\rangle$, as is common for fcc samples. They glide along $\langle 110\rangle$ directions and the generated stacking fault planes are $\{111\}$ planes. We note that the nucleation of dislocations in nanocrystalline samples at grain boundaries under stress does not always follow Schmid's law, as has been shown in several systematic studies $[49,50]$.

The number and length of dislocations (Fig. 4) are considerably higher in the coarse-grained sample. Furthermore, the region where dislocations are nucleated extends towards the upper end of the vice plates and even above the vice plates in the coarse-grained sample. Comparatively, the region where the dislocations are nucleated is concentrated towards the lower surface in the fine-grained sample. Not surprisingly, the largest amount of dislocation nucleation is found in the pressing phase, where a previously well-relaxed sample is quickly strained out of equilibrium. However, even the holding and release phases either produce novel dislocations or let already existing dislocations migrate. These findings corroborate the conclusion that dislocation plasticity is more readily found in the coarsegrained sample.
The role of grain-boundary activity can also be analyzed in Fig. 4. After pressing the samples, almost all grain boundaries in the bottom half of the sample are found to be strongly sheared, VMSS $>0.1$. Interestingly, the grains close to the vice plates are not as sheared as those in the middle of the samples. This is due to the fact that close to the plates, the stress field is mostly uniaxial, and more shear is expected at the upper ends of the plates and in the middle of the sample. Grain-boundary activities continue during the holding and the release phases, but are more prominent in the fine-grained sample.

Besides shear strain, hydrostatic pressure is an important indicator of the stress fields generated in the sample. Hydrostatic pressure is shown in Fig. 5 in atomic resolution; pressure data are averaged over $0.5 \mathrm{ps}$ to get rid of atomic vibrations. The plots give the same view as Fig. 4. As was noted above, the averaged (uniaxial) stress in the $x$ direction amounts to $2.5 \mathrm{GPa}$ in the bottom half of the sample. The figure shows that even in the bottom half of the sample, the hydrostatic pressure is far from a homogeneous distribution; this is caused by the differing orientations of the grains. While at the end of the pressing phase, the grain interiors are all compressively strained, some regions - in particular regions at grain boundaries - are under tensile strain. Grain boundaries act as sources of high stress; some low-angle grain boundaries- can be identified in this figure as a chain of dislocations with their alternating compressive and tensile stress fields. Other grain boundaries are under primarily compressive stress.

During the holding phase, pressures equilibrate somewhat in the grains, as can be seen by a stronger mix of compressive and tensile stress regions. This goes in line with the reduction of the forces needed to keep the vice plates in position (Fig. 2). After release, this equilibration process has further proceeded, since now the global pressure vanishes.

The final snapshots in Fig. 5 show the stresses remaining in the sample after the entire process; they constitute the residual stresses. A comparison with Fig. 4 demonstrates that some of the stresses inside the grains may be attributed to the dislocations that have been produced there; other sources of high pressure are in the vicinity of triple junctions of grain boundaries. However, the inhomogeneity of the pressure distribution - which is under vanishing global pressure - is astonishing, since pressures vary between -1 and $+1 \mathrm{GPa}$. The coarse-grained system shows stronger residual stresses at some of the grain boundaries than the fine-grained system.

In summary, Figs. 4 and 5 demonstrate that dislocation plasticity is more active in the coarse-grained sample, while grain-boundary processes are important for both samples studied. Defects in the sample - here in particular, grain boundaries - act as stress concentrators and are also the places where residual stresses build up. 


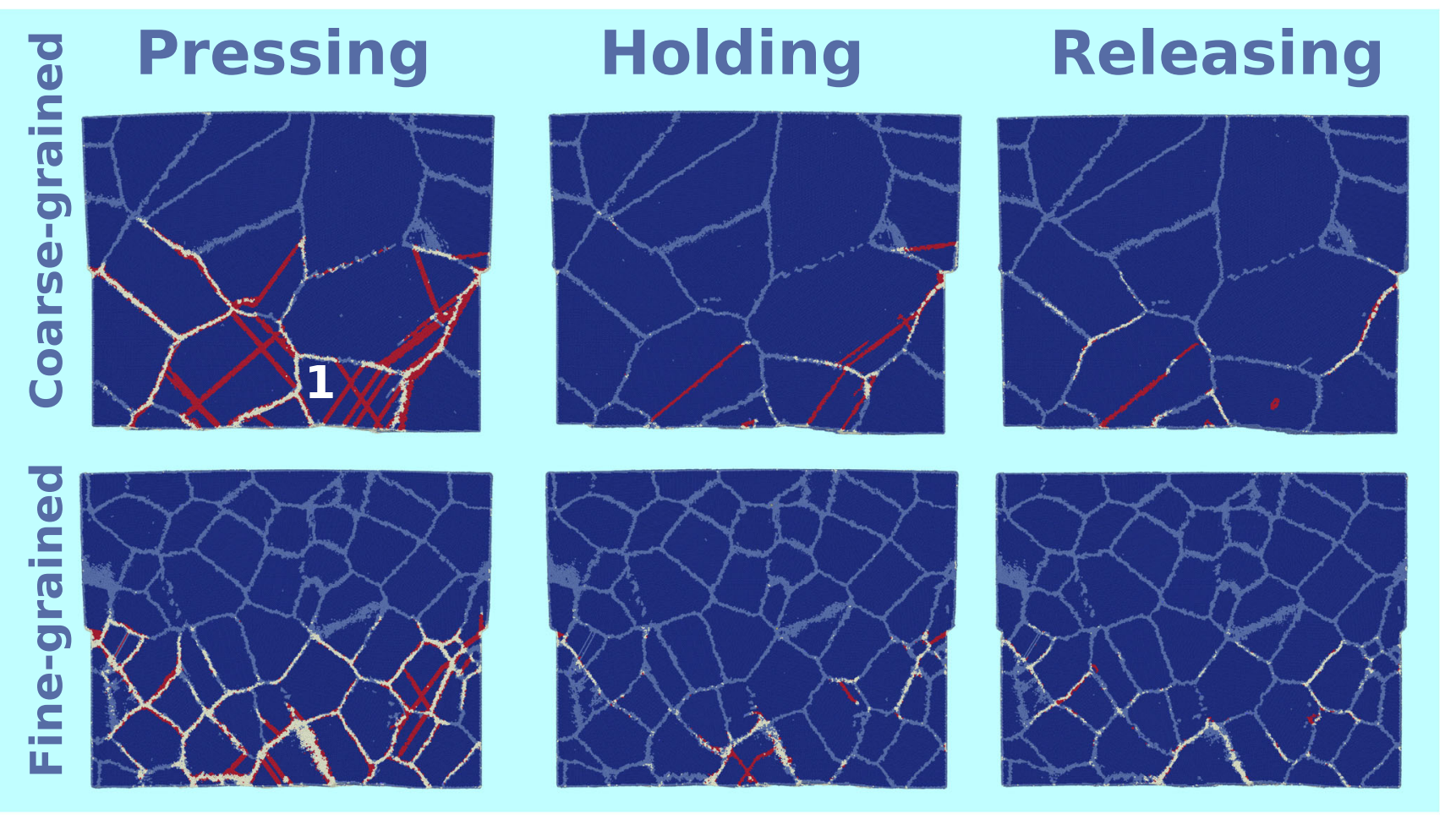

Fig. 4 VMSS map of coarse-grained and fine-grained samples, during pressing, holding and release. The reference configurations are the same as in Fig. 3. In this side view on the sample, dark blue represents atoms in fcc lattice structure inside the grains and light blue represents atoms at grain boundaries. White characterizes grain-boundary atoms with VMSS $>0.1$, and red atoms have VMSS $>0.1$ and belong to the grain interior. The grain in the coarse-grained sample denoted by "1" serves to discuss dislocation contributions to surface roughness in Section 3.3

\subsection{Surface roughness}

The snapshots, Figs. 4 and 5, show already strong deformations of the sample surface. During pressing and holding, the upper surface becomes curved in a convex way; this curvature disappears after release. The lower surface becomes rough, and this roughness does not disappear. This is due to the different grain orientations and the various grain rotations (and displacement processes). Another interesting feature is the left-right asymmetry, which develops for the coarse-grained sample in the pressing phase and remains through the holding and release phases and will be discussed in Sections 3.4 and 3.5 below.

We analyze the roughness developing in the bottom surface in greater detail in Fig. 6 which shows the height distribution of the bottom surface profile for the coarsegrained and fine-grained samples. The average and the standard deviation of the distributions are assembled in Table 1. We use the standard deviation of the height profile as a measure of the surface roughness.

During pressing, the standard deviation increases by a factor of 4.5 (7) with respect to the original value for the coarse-grained (fine-grained) sample. During holding, the roughness increases even further, but reduces somewhat in the release phase. The reason for this surface roughness is grain displacement and rotation and will be discussed in the next two sections. These processes cannot be undone during the release phase. The height profile of the coarse-grained sample (Fig. 6a) differs significantly from a Gaussian profile as it exhibits two peaks in the height spectrum, which are caused by individual grains popping out of the original surface. This feature is even enhanced during the holding phase. There are 42 grains in the fine-grained sample at the bottom surface, but only 13 grains in the coarse-grained sample. Therefore, the multitude of grains contributing to the surface in the fine-grained sample randomize the height profile and lead to a distribution that is closer to a Gaussian profile.

In addition, dislocations contribute to surface roughness. This can be clearly observed in the grain denoted by " 1 " in the coarse-grained sample in Fig. 4. This grain has a large area on the lower surface and dislocations absorbed on it lead to surface steps which contribute considerably to the surface roughness of this grain. 


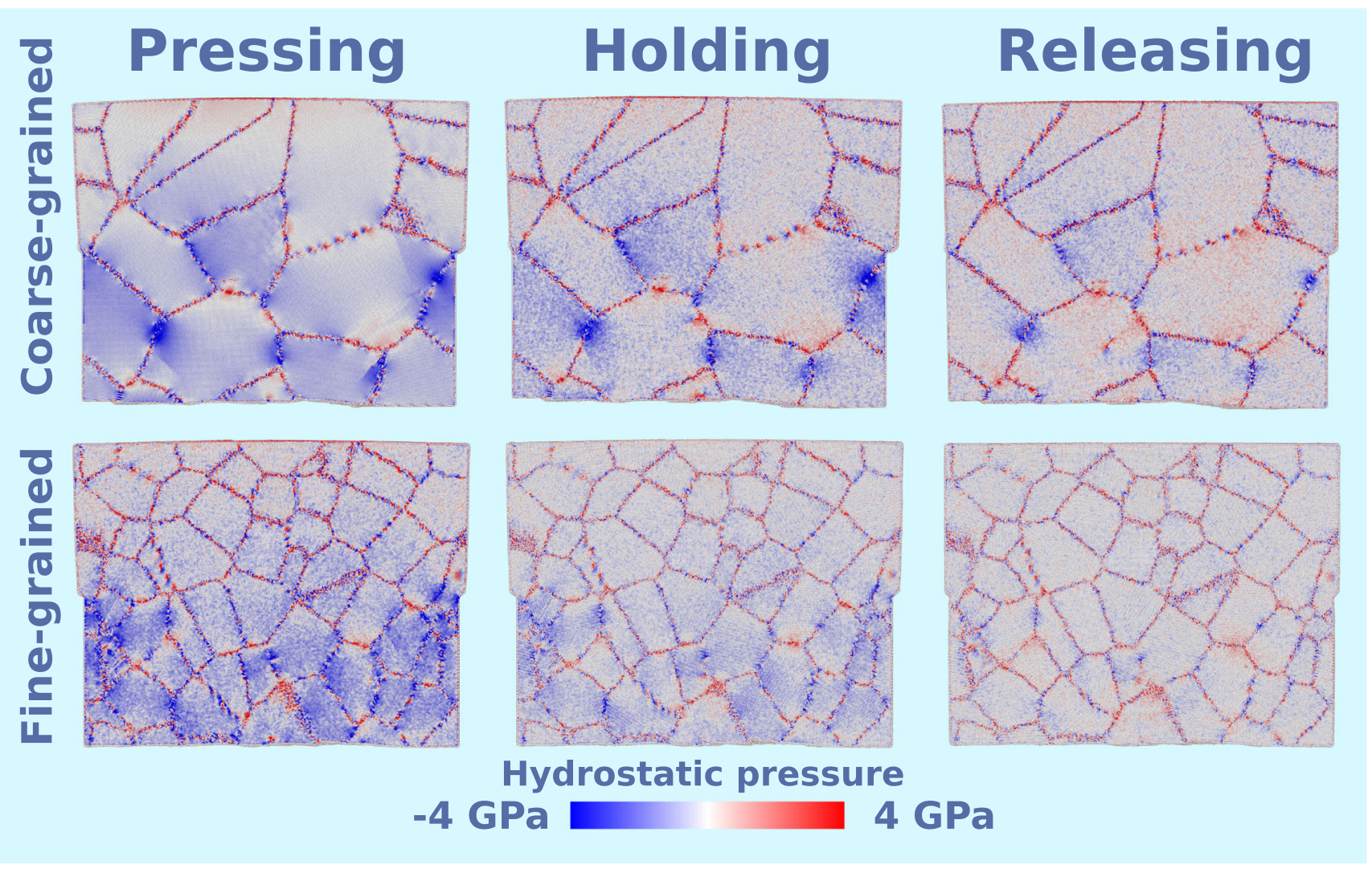

Fig. 5 Hydrostatic pressure. Negative values denote compressive pressure

\subsection{Center-of-mass grain displacement}

In order to describe the translational motion of grains, we study their center-of-mass displacements, $\vec{d}$ [27]. Since there is no standard analysis available for this purpose, we proceed as follows. We cut 7 slabs extending in the $x-z$ plane with a thickness of $2 \mathrm{~nm}$ in the $y$ direction. The slabs are evenly distributed on the $y$ axis. In these slabs it is possible to analyze the center-of-mass displacement of the grains in the $x-z$ plane, as we can neglect any motion in $y$ direction. We present the resulting translational motions as histograms in Fig. 7. This figure shows the displacements during the pressing, holding and release phases. In each phase, the displacement between the beginning and the end of the phase are shown. The total displacement, $d_{\mathrm{abs}}=|\vec{d}|$, and the components $d_{x}$ and $d_{z}$ along the $x$ and $z$ axes are also shown.

Grain displacements during the pressing phase (Fig. 7a) are best described by the component parallel to the force axis, $d_{x}$. The distributions show two peaks at $-20 \AA$ and $+20 \AA$, corresponding to grains located close to the vice plates, which are forced to move the entire distance covered by the nearby vice. The majority of the grains, however, suffer only little motion, since they are located in the middle of the sample, between the two vices. Apart from this horizontal motion, a component $d_{z}$ in vertical direction is seen. The majority of the grains are moved upwards and thus contribute to the curvature of the upper surface seen in Fig. 6. In the pressing phase, no large difference between the coarse-grained and the fine-grained samples shows up.

Note that in a purely elastic compression, grain centers initially positioned at $x$ would move by a distance $(1-\varepsilon) x$, where $\varepsilon=0.05$ is the applied uniaxial strain. The $d_{x}$ data in Fig. 7a would thus follow a rectangular distribution of constant height. The simulation results thus demonstrate the large deviation of plastic grain flow from this elastic reference case.

Considerable differences are observed during the holding phase (Fig. 7b). Here, grains in the coarse-grained sample are shifted to the right-hand side, $d_{x}>0$, with an amplitude of $5 \AA$. This shift corresponds to the left-right asymmetry shown in Figs. 4 and 5. The fine-grained sample also drifts further in horizontal direction in order to release the compressive strain present in the lower part of the sample; on the other hand, grain movements in the coarse-grained sample are larger in size, but show as much upwards as downwards motion.

The left-right asymmetry showing up here is caused by the individual grain movements. In the coarse-grained sample, an analysis of grain motion shows a relative movement 

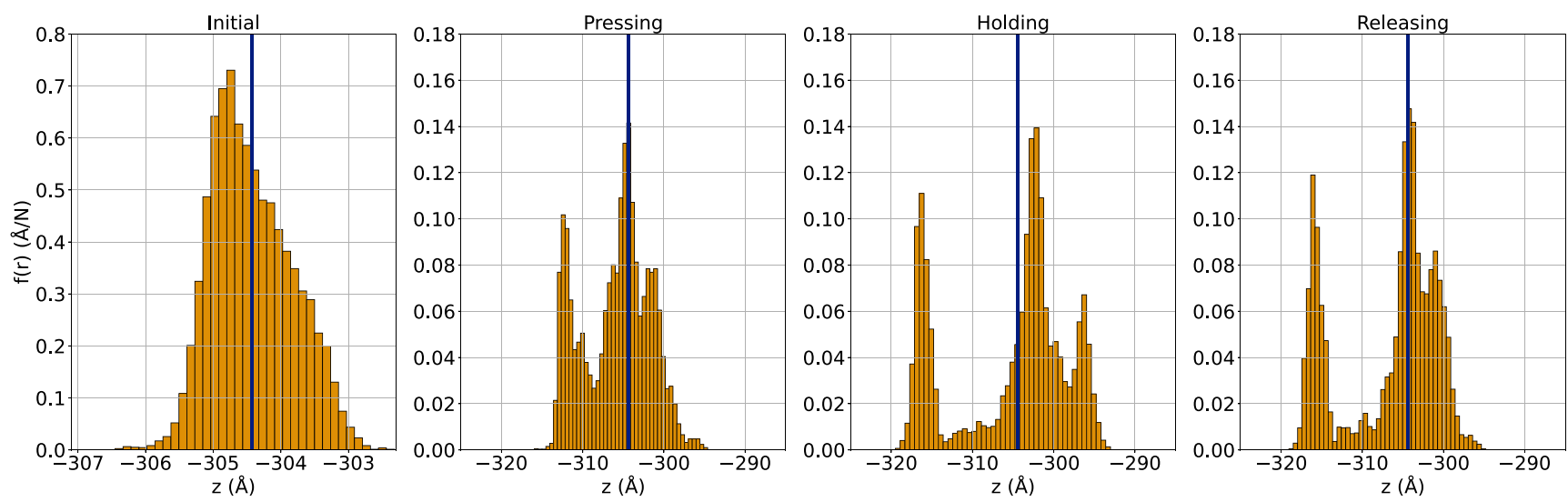

(a)
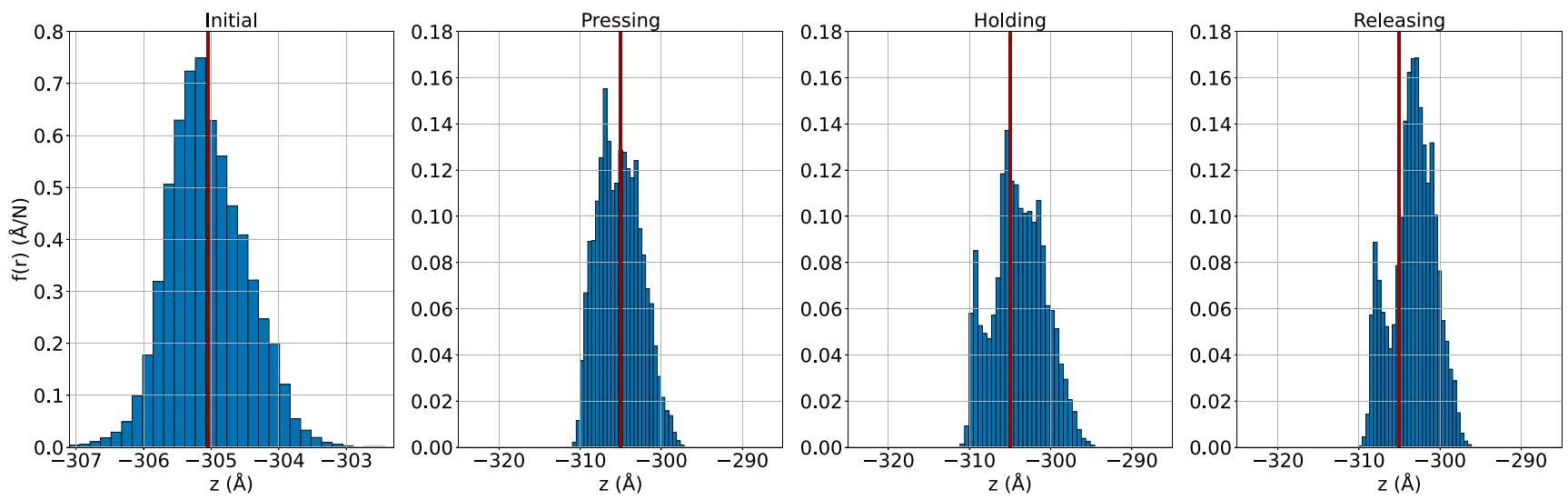

(b)

Fig. 6 Height profile of the bottom sample surface for the $\mathbf{a}$ coarse-grained sample and $\mathbf{b}$ fine-grained sample. The vertical line indicates the original average surface position. Note the change in scale between the initial and the deformed profiles

of the upper part of the sample towards the right, accompanied by a downward motion of the grains in the vicinity of the right-hand vice plate. The fine-grained sample does not show a corresponding asymmetry, since here, grain motion is more random.

Also during the release phase, considerable grain movements are observed (Fig. 7c). The curvature of the upper part of the sample is reduced, leading to a downward grain motion, $d_{z}<0$. While the grain motion in the $x$ direction is symmetrical for the fine-grained sample, the coarse-grained sample tends to reduce the asymmetric motion that occurred during the holding phase, featuring an average $d_{x}<0$.

The asymmetry observed in the grain motion for the coarse-grained sample also shows up when considering the forces acting on the vices (Fig. 2). As the vices are compressed with constant velocity, individual grain movements oppose the vice motion with different forces. Interestingly, this asymmetry is particularly pronounced in the holding stage, but is also visible in the pressing and release stages.

Table 1 Average (avg) and standard deviation (std) of the surface profiles (Fig. 6)

\begin{tabular}{|c|c|c|c|c|c|c|c|c|}
\hline \multirow[t]{2}{*}{ Sample } & \multicolumn{2}{|l|}{ Initial } & \multicolumn{2}{|l|}{ Pressing } & \multicolumn{2}{|l|}{ Holding } & \multicolumn{2}{|l|}{ Release } \\
\hline & avg & std & avg & std & avg & std & avg & std \\
\hline Coarse-grained & -304.42 & 0.60 & -305.85 & 4.15 & -305.30 & 7.12 & -306.61 & 5.94 \\
\hline Fine-grained & -305.05 & 0.60 & -305.13 & 2.62 & -303.97 & 3.21 & -303.31 & 2.63 \\
\hline
\end{tabular}

Data are in $\AA$ units 


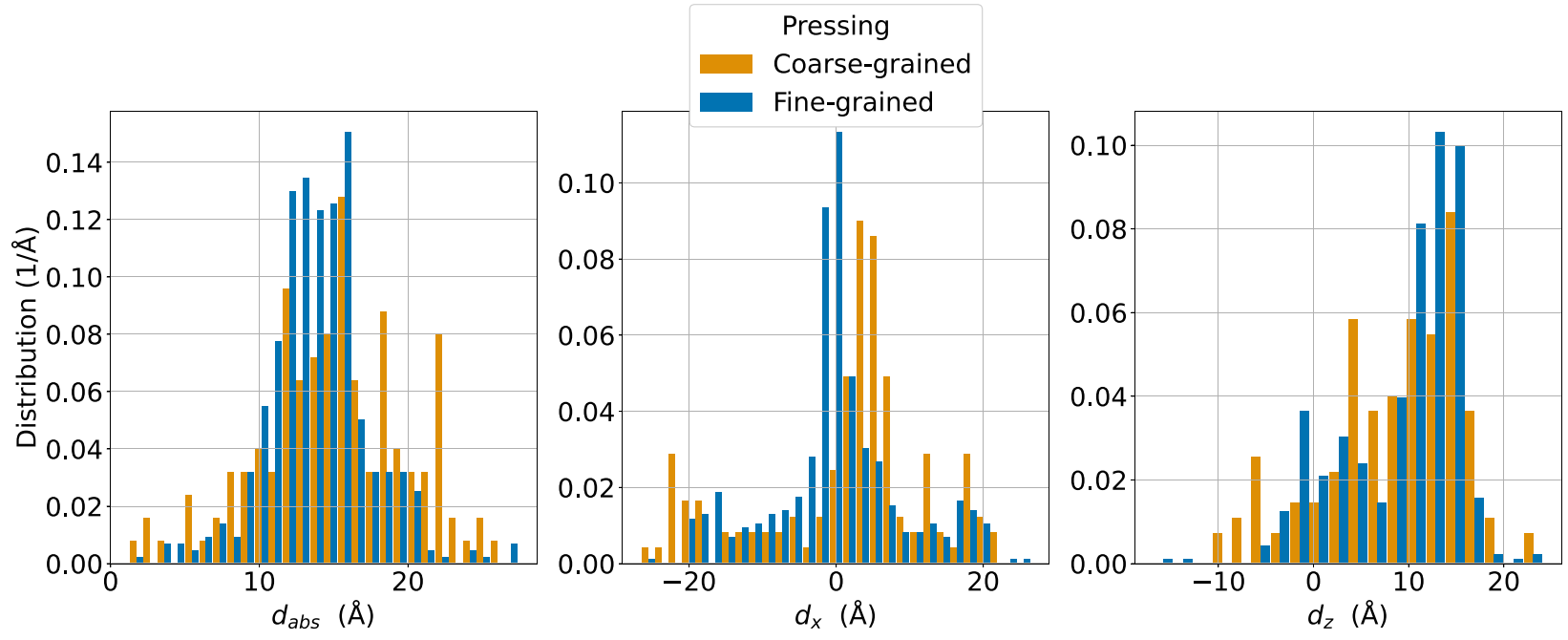

(a)
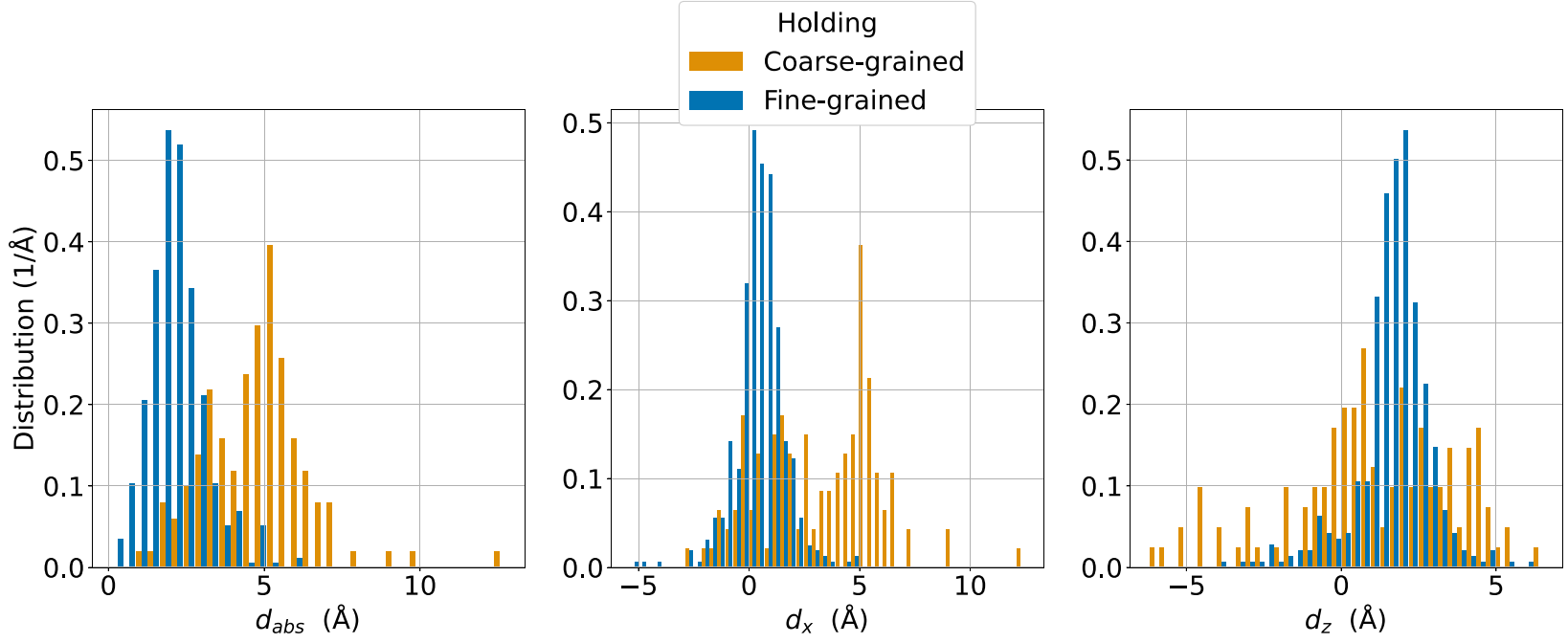

(b)
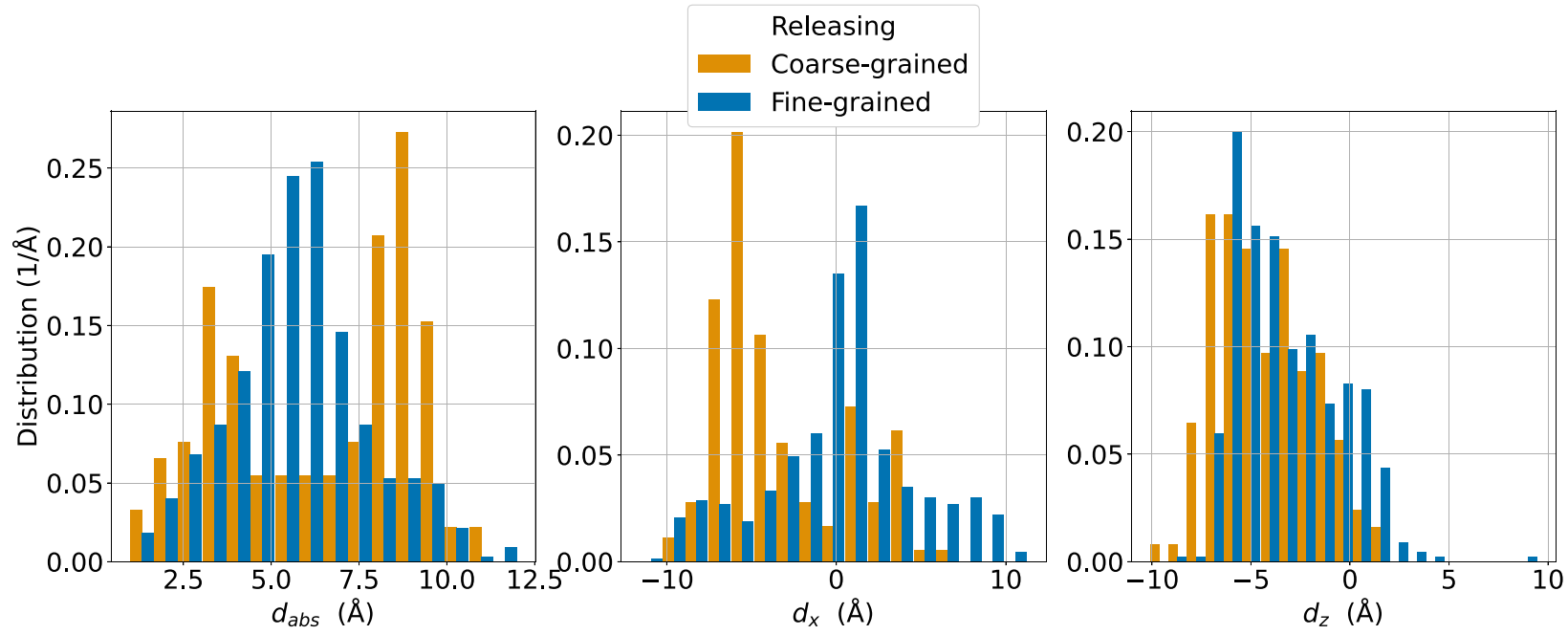

(c)

Fig. 7 Normalized distributions of the center-of-mass displacements of grains during the a pressing, $\mathbf{b}$ holding, and $\mathbf{c}$ release phase 
Figure 8 gives an overview over the magnitude and direction of the center-of-mass translation vectors during the pressing phase for the fine-grained sample. A clear flow pattern is established, in which the inward moving vice plates let the grains move inward along the $x$ axis. When these flows converge in the middle of the sample, the grains move upwards along the $z$ axis. In the upper half of the sample, above the vice plates, the flow is everywhere in the $z$ direction, leading to sample expansion in this direction.

We conclude that grain motion is mainly governed by the movement of the vices and by the upwards expansion of the sample. The coarse-grained sample shows more individuality in grain motions such that an asymmetry with respect to the behavior of the left- and right-hand side begins to appear. In contrast, the grain motion of the fine-grained sample is more randomized and does not show this asymmetry.

\subsection{Grain rotation}

In the course of pressing, grains are not only translated, but also rotated. We calculate the rotation angle by using a quaternion formulation to identify rotation, which transforms the original orientation of each grain to the final orientation after pressing [51-53].

Figure 9 shows the distribution of the rotation angles during the pressing phase. Quite large angles of $8.5^{\circ}$ are observed with an average value of $1.29^{\circ}\left(1.40^{\circ}\right)$ for the coarse-grained (fine-grained) sample. The angles are similar for the 2 samples; however, the largest angles occur for the fine-grained sample. The effect of the grain rotation is the tilting of the grains at the lower sample surface which is visible in Figs. 4 and 5 and leads to the sample roughness discussed in Section 3.3. During the holding and

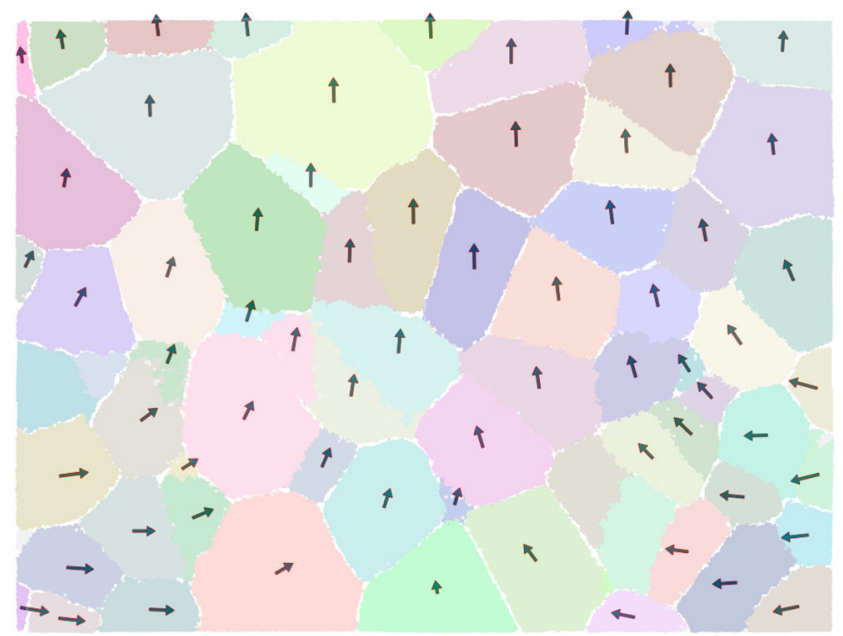

Fig. 8 Center-of-mass grain displacement during the pressing stage in the fine-grained sample, overlaid with the initial grain structure. The arrows point from the original position of the center of mass to its final position

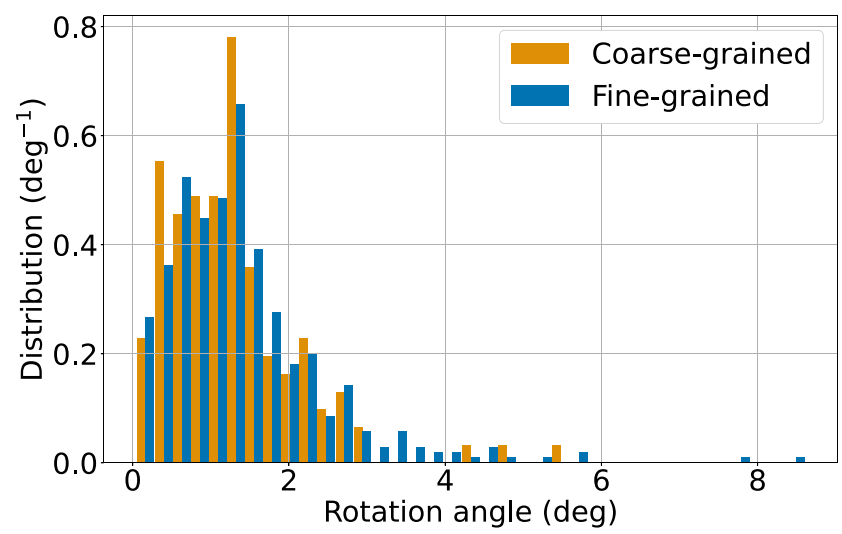

Fig. 9 Distribution of grain rotation angles during the pressing phase

release phase, rotation angles were $<0.01^{\circ}$, indicating that grains do not rotate back towards their initial orientations. This demonstrates that sizable rotations only occur under immediate sample loading.

\subsection{Sample distortion}

The length changes of the sample after release from the fixture are called distortions. We discuss here these length changes in all phases during and after the loading process. The length changes are measured in the vertical direction at 3 positions $\mathrm{A}, \mathrm{B}, \mathrm{C}$ at the left, middle and right of the sample, and at 3 positions in the horizontal direction (D, E, F) in the bottom, middle, and top of the sample (see Fig. 10a). In detail, due to the atomistic roughness of the samples, for the example of position A, we measure the average height of the sample in a slab of width $1 \mathrm{~nm}$ extending at a distance of $3 \mathrm{~nm}$ from the lateral surface in the $z-y$ plane; this averaging process is performed because of the roughness of the samples due to their nanocrystalline structure (cf. Section 3.3).

Figure 10 shows the distortions measured as fractional changes of the lengths with respect to their initial values. The sample shrank horizontally under the compressive loading by the vice plates. These induce a compressive strain of $-5 \%$ which exactly equals the distortion measured at $\mathrm{D}$ in the pressing and holding phase. After release, the sample does not return to its original length, since the deformation was plastic; the final distortion amounts to $-3.8(-2.7) \%$ for the coarse-grained (fine-grained) sample. Horizontal distortions in the middle of the sample at position $\mathrm{E}$ are considerably reduced, and at the top of the sample, position $\mathrm{F}$, the sample expands in all phases. This expansion is caused by material flow from the lower half of the sample towards the upper half of the sample.

The horizontal narrowing of the sample is accompanied by an expansion in the vertical direction. This expansion is more or less uniform for the fine-grained sample, but 


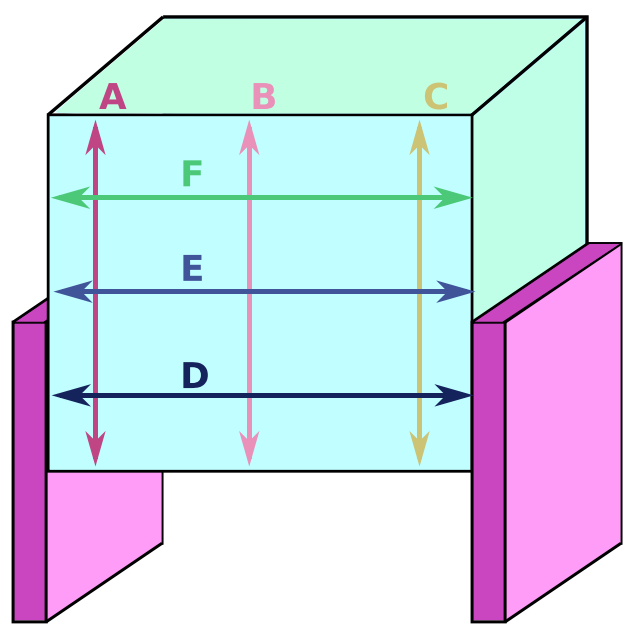

(a)

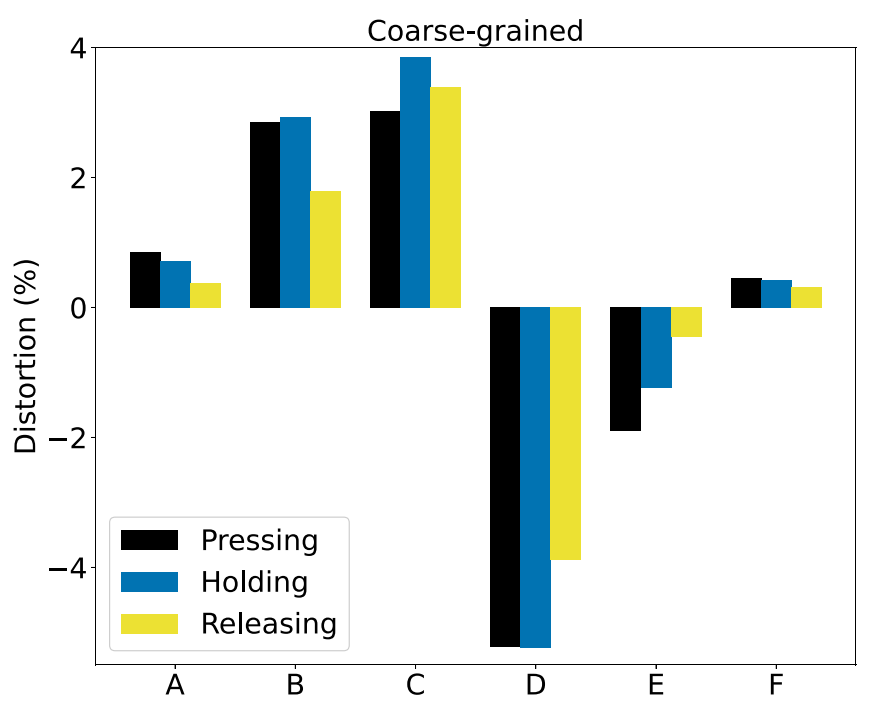

(b)

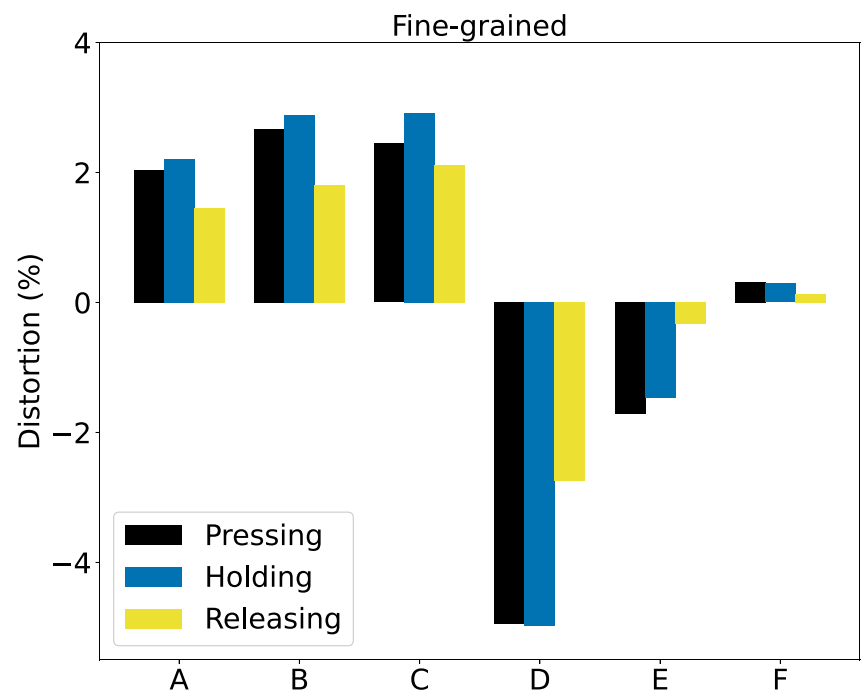

(c)

Fig. 10 a Schematics indicating the positions where vertical $(\mathrm{A}-\mathrm{C})$ and horizontal $(\mathrm{F}-\mathrm{D})$ sample distortions were measured. $\mathbf{b}$ and $\mathbf{c}$ show simulation results for distortions for the coarse-grained (b) and fine-grained (c) sample in the pressing, holding and release phase

shows a considerable left-right asymmetry for the coarsegrained sample, which was already observed and discussed previously (Section 3.4).

We conclude that sample distortions after release are stronger for the coarse-grained than for the fine-grained sample. This is caused by the easier grain movement of the large grains, as evidenced in Section 3.4.

Compared to real applications of nanomanufacturing, the model sample has a larger surface-to-volume ratio. Therefore, it can be assumed that surface effects are less pronounced in real applications. With a higher grain number in real samples, processes in a large number of grains will add up to the total distortion such that the effects in individual grains — while still present — will lose importance for the average distortion in the sample, since variations between individual grains might smoothen out.

\section{Summary}

This study monitored the plastic deformation processes occurring in a polycrystalline Al bar under external inhomogeneous loading, representing vice plates. The processes occurring in the pressing, the holding and the release phases were analyzed and gave the following results.

1. Fine-grained material (grain size of $10 \mathrm{~nm}$ ) showed only little dislocation activity inside the grains during 
plastic deformation, while dislocation activity is strong in the deformation of coarse-grained material (grain size of $18 \mathrm{~nm}$ ). This result extends previous studies of the deformation of nanocrystalline fcc metals [13, 14, 17, 45, 47], which were mainly based on homogeneous strains in bulk samples, to the case where inhomogeneous strains and free surfaces are relevant.

2. We followed the grain displacements by monitoring their center-of-mass trajectories. The results show strong deviations from a simple affine compaction of the material; rather, grains move in a strongly inhomogeneous way.

3. As a consequence of the nanometric size of the investigated Al bar, grain motions are not symmetric with respect to the bar center, but exhibit a left-right asymmetry. It is caused by grain displacement and rotation and occurs pronouncedly in the coarse-grained sample, while it averages out in the fine-grained sample. It may be expected that this asymmetry will decrease for larger samples.

4. The grains also suffer considerable rotations with an average rotation angle of $1.3^{\circ}-1.4^{\circ}$. These grain rotations only occurred in the pressing phase and not during holding or releasing.

5. These grain rotations lead to a considerable roughening of the bottom surface of the sample, which does not vanish after releasing the vice fixtures.

6. The residual stresses in the material resulting from dislocation activity and grain-boundary processes build up in the pressing phase and do not relax during the holding and release phases.

7. Due to the plastic deformations occurring, the sample shows a distortion after the process. The final distortion at the position where the vice plates acted amounts to around half the applied strain, but decreases in other areas. In the direction perpendicular to the applied strain, the sample grows. The coarse-grained sample shows higher distortion values than the fine-grained sample because of longer-ranged grain displacements.

Our results show that the final sample distortion is based on plastic processes that are mediated both by dislocation glide and grain displacement and rotation. In future work, the results presented here should be extended to other material classes. Besides bcc metals, such as $\mathrm{Fe}$, the distortion processes in alloys would be particularly relevant.

Acknowledgements Simulations were performed at the High Performance Cluster Elwetritsch (RHRK, TU Kaiserslautern, Germany).

Funding Open Access funding enabled and organized by Projekt DEAL. This work received support from the Deutsche Forschungsgemeinschaft (DFG, German Research Foundation) - project number 252408385 - IRTG 2057.
Availability of data and material This study is based only on data obtained using the methods described in this paper.

Code availability The simulations were performed by the open-source code LAMMPS available under http://lammps.sandia.gov/ [36].

\section{Declarations}

Conflict of interest The authors declare no competing interests.

Open Access This article is licensed under a Creative Commons Attribution 4.0 International License, which permits use, sharing, adaptation, distribution and reproduction in any medium or format, as long as you give appropriate credit to the original author(s) and the source, provide a link to the Creative Commons licence, and indicate if changes were made. The images or other third party material in this article are included in the article's Creative Commons licence, unless indicated otherwise in a credit line to the material. If material is not included in the article's Creative Commons licence and your intended use is not permitted by statutory regulation or exceeds the permitted use, you will need to obtain permission directly from the copyright holder. To view a copy of this licence, visit http://creativecommons. org/licenses/by/4.0/.

\section{References}

1. Dieter GE (1986) Mechanical metallurgy, 3rd ed. McGraw-Hill

2. Weber D, Kirsch B, D'Elia CR, Linke BS, Hill MR, Aurich JC (2019) Concept to analyze residual stresses in milled thin walled monolithic aluminum components and their effect on part distortion. In: Wulfsberg JP, Hintze W, Behrens B-A (eds) Production at the leading edge of technology. Springer, Berlin, pp 287-296

3. Yuan F, Wu X (2013) Size effects of primary/secondary twins on the atomistic deformation mechanisms in hierarchically nanotwinned metals. J Appl Phys 113:203516

4. D’Alvise L, Chantzis D, Schoinochoritis B, Salonitis K (2015) Modelling of part distortion due to residual stresses relaxation: An aeronautical case study. Procedia CIRP 31:447-452.

5. Ke X, Ye J, Pan Z, Geng J, Besser MF, Qu D, Caro A, Marian J, Ott RT, Morris Wang Y, Sansoz F (2019) Ideal maximum strengths and defect-induced softening in nanocrystalline-nanotwinned metals. Nat Mater 18:1207-1214

6. Aurrekoetxea M, López de Lacalle LN, Llanos I (2020) Machining stresses and initial geometry on bulk residual stresses characterization by on-machine layer removal. Materials 13:1445

7. Fan Z, Cao L, Liu F (2020) FEM analysis of the distortion of thinwalled sealing part affected by the machining-induced residual stress. IOP Conf Ser Mater Sci Eng 768:042028

8. Aurich JC, Carrella M, Walk M (2015) Micro grinding with ultra small micro pencil grinding tools using an integrated machine tools. CIRP Ann Manuf Technol 64:325-328

9. Bhushan B (ed) (2017) Springer Handbook of Nanotechnology, 4th edn. Springer, Heidelberg

10. Fang FZ, Zhang XD, Gao W, Guo YB, Byrne G, Hansen HN (2017) Nanomanufacturing - perspective and applications. CIRP Ann 66:683-705

11. Sinnott SB, Heo S-J, Brenner DW, Harrison JA, Irving DL (2010) Computer simulations of nanometer-scale indentation and friction. In: Bhushan B (ed) Springer Handbook of Nanotechnology, chapter 31. 3rd edn. Springer, pp 955-1012 
12. Gerberich W, Tadmor EB, Kysar J, Zimmerman JA, Minor AM, Szlufarska I, Amodeo J, Devincre B, Hintsala E, Ballarini R (2017) Review article: Case studies in future trends of computational and experimental nanomechanics. Jl Vacuum Sci Technol A: Vacuum Surf Films 35:060801

13. Schiøtz J, Di Tolla FD, Jacobsen KW (1998) Softening of nanocrystalline metals at very small grain sizes. Nature 391:561-563

14. Van Swygenhoven H, Weertman JR (2006) Deformation in nanocrystalline metals. Mater Today 9:24-31

15. Shimokawa T, Nakatani A, Kitagawa H (2005) Grain-size dependence of the relationship between intergranular and intragranular deformation of nanocrystalline al by molecular dynamics simulations. Phys Rev. B 71:224110

16. Huang H, Van Swygenhoven H (2009) Atomistic simulations of mechanics of nanostructures. MRS Bull 34:160

17. Li X, Wei Y, Yang W, Gao H (2009) Competing grain-boundaryand dislocation-mediated mechanisms in plastic strain recovery in nanocrystalline aluminum. Proc Natl Acad Sci 106:16108-16113

18. Zepeda-Ruiz LA, Stukowski A, Oppelstrup T, Bulatov VV (2017) Probing the limits of metal plasticity with molecular dynamics simulations. Nature 550:492

19. Zhou K, Liu B, Shao S, Yao Y (2017) Molecular dynamics simulationsMolecular dynamics simulations of tension-compression asymmetry in nanocrystalline copper. Phys Lett A 381:1163-1168

20. Zhou X, Feng Z, Zhu L, Xu J, Miyagi L, Dong H, Sheng H, Wang Y, Li Q, Ma Y, Zhang H, Yan J, Tamura N, Kunz M, Lutker K, Huang T, Hughes DA, Huang X, Chen B (2020) High-pressure strengthening in ultrafine-grained metals. Nature 579:67-72

21. Huang CC, Chiang TC, Fang TH (2015) Grain size effect on indentation of nanocrystalline copper. Appl Surf Sci 353:494-498

22. Ruestes CJ, Bringa EM, Gao Y, Urbassek HM (2017) Molecular dynamics modeling of nanoindentation. In: Tiwari A, Natarajan S (eds) Applied Nanoindentation in Advanced Materials, chapter 14. Wiley, Chichester, pp 313-345

23. Alabd Alhafez I, Brodyanski A, Kopnarski M, Urbassek HM (2017) Influence of tip geometry on nanoscratching. Tribol Lett 65:26

24. Gao Y, Urbassek HM (2014) Evolution of plasticity in nanometric cutting of Fe single crystals. Appl Surf Sci 317:6-10

25. Shimada S, Ikawa N, Tanaka H, Uchikoshi J (1994) Structure of micromachined surface simulated by molecular dynamics analysis. CIRP Ann 43:51-54

26. Meyers MA, Chawla KK (2010) Mechanical behavior of materials, 2nd ed. Cambridge University Press, Cambridge

27. Farkas D, Mohanty S, Monk J (2008) Strain-driven grain boundary motion in nanocrystalline materials, Materials Science and Engineering A 493:33-40.

28. Vo NQ, Averback RS, Bellon P, Odunuga S, Caro A (2008) Quantitative description of plastic deformation in nanocrystalline $\mathrm{Cu}$ : Dislocation glide versus grain boundary sliding. Phys Rev B 77:134108

29. Zaafarani N, Raabe D, Roters F, Zaefferer S (2008) On the origin of deformation-induced rotation patterns below nanoindents. Acta Mater 56:31

30. Romero PA, Järvi TT, Beckmann N, Mrovec M, Moseler M (2014) Coarse graining and localized plasticity between sliding nanocrystalline metals. Phys Rev Lett 113:036101

31. Garcia DR, Zhang Z, Linke BS, Urbassek HM (2018) Molecular dynamics simulations of single grain pure aluminum in a vice fixture for nanomanufacturing applications. CIRP J Manuf Sci Technol 23:91-97

32. Voronoi G (1908) Nouvelles applications des paramètres continus à la théorie des formes quadratiques. Reine Angew Math 134:199

33. Hirel P (2015) Atomsk: A tool for manipulating and converting atomic data files. Comput Phys Commun 197:212-219

34. Prakash A, Hummel M, Schmauder S, Bitzek E (2016) Nanosculpt: A methodology for generating complex realistic configurations for atomistic simulations. MethodsX 3:219-230

35. Mishin Y, Farkas D, Mehl MJ, Papaconstantopoulos DA (1999) Interatomic potentials for monoatomic metals from experimental data and ab initio calculations. Phys Rev B 59:3393

36. Plimpton ST (1995) Fast parallel algorithms for short-range molecular dynamics. J Comput Phys 117:1-19. http://lammps. sandia.gov/

37. Stukowski A (2010) Visualization and analysis of atomistic simulation data with OVITO - the Open Visualization Tool. Model Simul Mater Sci Eng 18:015012. http://www.ovito.org/

38. Zhang Z, Urbassek HM (2018) Indentation into an Al-Si composite: Enhanced dislocation mobility at interface. J Mater Sci 53:799-813

39. Bitzek E, Derlet PM, Anderson PM, Van Swygenhoven H (2008) The stress-strain response of nanocrystalline metals: A statistical analysis of atomistic simulations. Acta Mater 56:4846-4857

40. Dupont V, Sansoz F (2008) Quasicontinuum study of incipient plasticity under nanoscale contact in nanocrystalline aluminum. Acta Mater 56:6013-6026

41. Kadau K, Germann TC, Lomdahl PS, Holian BL, Kadau D, Entel P, Kreth M, Westerhoff F, Wolf DE (2004) Molecular-dynamics study of mechanical deformation in nano-crystalline aluminum. Metall Mater Trans A 35:2719

42. Prakash A, Weygand D, Bitzek E (2017) Influence of grain boundary structure and topology on the plastic deformation of nanocrystalline aluminum as studied by atomistic simulations. Int J Plast 97:107-125

43. Mahata A, Zaeem MA (2019) Evolution of solidification defects in deformation of nano-polycrystalline aluminum. Comput Mater Sci 163:176-185

44. Lund AC, Schuh CA (2005) Strength asymmetry in nanocrystalline metals under multiaxial loading. Acta Mater 53:3193-3205

45. Schiøtz J (2004) Atomic-scale modeling of plastic deformation of nanocrystalline copper. Script Mater 51:837-841.

46. Schiøtz J, Vegge T, Di Tolla FD, Jacobsen KW (1999) Atomic-scale simulations of the mechanical deformation of nanocrystalline metals. Phys Rev B 60:11971

47. Schiøtz J, Jacobsen KW (2003) A maximum in the strength of nanocrystalline copper. Science 301:1357-1359

48. Gupta A, Gruber J, Rajaram SS, Thompson GB, McDowell DL, Garritt J (2020) Tucker, On the mechanistic origins of maximum strength in nanocrystalline metals. npj Comput Mater 6:153

49. Tschopp MA, Tucker GJ, McDowell DL (2008) Atomistic simulations of tension-compression asymmetry in dislocation nucleation for copper grain boundaries. Comput Mater Sci 44:351-362

50. Wyman RD, Fullwood DT, Wagoner RH, Homer ER (2017) Variability of non-schmid effects in grain boundary dislocation nucleation criteria. Acta Mater 124:588-597

51. Barrales Mora LA (2008) 2D and 3D Grain Growth Modeling and Simulation, Ph.D. thesis, RWTH Aachen

52. El Shawish S, Cizelj L (2016) Numerical investigation of grain misorientations at and close to the free surface of fcc polycrystalline metals. Comput Mater Sci 113:133-142

53. Kasemer M, Zepeda-Alarcon E, Carson R, Dawson P, Wenk H-R (2020) Deformation heterogeneity and intragrain lattice misorientation in high strength contrast, dual-phase bridgmanite/periclase. Acta Mater 189:284-298

Publisher's note Springer Nature remains neutral with regard to jurisdictional claims in published maps and institutional affiliations. 